\title{
Risk of Thrombus Fragmentation during Endovascular Stroke Treatment
}

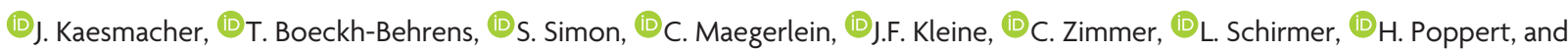 \\ (D)T. Huber

\section{ABSTRACT}

BACKGROUND AND PURPOSE: Periprocedural thrombus fragmentation is a relevant risk in endovascular stroke treatment. Because factors influencing its occurrence are largely unknown, this study addresses a potential relationship between thrombus histology and clot stability.

MATERIALS AND METHODS: Eighty-five patients with anterior circulation stroke treated with thrombectomy were included in this retrospective study. The number and location of emboli after retrieving the primary thrombus, the number of maneuvers, and TICl scores were evaluated. H\&E and neutrophil elastase staining of retrieved clots was performed, and semiquantitative measurements of thrombus components were correlated with procedural parameters.

RESULTS: An inverse correlation between maneuvers required for thrombus retrieval and the number of distal and intermediate emboli was observed (Spearman $r,-0.23 ; P=.032$ ). Younger patients were at higher risk for periprocedural thrombus fragmentation (Spearman $r,-0.23 ; P=.032$ ). Bridging thrombolysis tended to be associated with fewer maneuvers ( 2 vs $3, P=.054)$ but more emboli $(1 \mathrm{vs} 0, P=.067)$. While no consistent correlation between procedural parameters and red/white blood cells and fibrin-/platelet fractions could be found, higher amounts of neutrophil elastase-positive cells within the thrombus were independently associated with the occurrence of multiple emboli (adjusted OR, 4.6; 95\% Cl, 1.1-19.7; $P=.041$ ) and lower rates of complete recanalization (adjusted OR, 0.3; 95\% Cl, 0.1-0.9; $P=.050$ ).

CONCLUSIONS: Younger age, easy-to-retrieve thrombi, and bridging thrombolysis may be risk factors for periprocedural thrombus fragmentation. Findings from standard histologic stains did not provide insight into thrombectomy-relevant thrombus stability. However, higher neutrophil levels in the thrombus tissue were related to an increased risk of periprocedural thrombus fragmentation. This observation aligns with the proposed thrombolytic capacity of neutrophil elastase and points to its potential clinical relevance in the context of stroke thrombectomy.

ABBREVIATIONS: $\mathrm{ACA}=$ anterior cerebral artery; $\mathrm{F} / \mathrm{P}=$ fibrin-/platelet accumulations; IQR $=$ interquartile range; $\mathrm{MT}=$ mechanical thrombectomy, $\mathrm{NE}=$ neutrophil elastase; $\mathrm{POS}=$ primary occlusion site; PTF = periprocedural thrombus fragmentation; RBC = red blood cells; $\mathrm{WBC}=$ white blood cells

M echanical thrombectomy (MT) of large-vessel occlusion has evolved as a safe and effective procedure that plays an indispensable role in modern therapeutic management of acute ischemic stroke. ${ }^{1-5}$ In recent randomized trials, high rates of suc-

Received September 12, 2016; accepted after revision December 12.

From the Departments of Neuroradiology (J.K., T.B.-B., C.M., J.F.K., C.Z., T.H.) and Neurology (S.S., L.S., H.P.), Klinikum rechts der Isar, Technical University of Munich, Munich, Germany.

Paper previously presented at: World Stroke Congress, October 26-29, 2016; Hyderabad, India.

Please address correspondence to Thomas Huber, MD, Department of Neuroradiology, Klinikum rechts der Isar, Technical University of Munich, Ismaninger Str 22, 81675 Munich, Germany; e-mail: Thomas-huber@tum.de

$\equiv$ Indicates article with supplemental on-line table.

Indicates article with supplemental on-line photos.

http://dx.doi.org/10.3174/ajnr.A5105 cessful recanalization (range, 59\%-88\%) were considered a key element in achieving excellent rates of good functional outcome (range, 33\%-71\%). ${ }^{6}$ However, not all successfully treated patients showed complete (TICI 3) recanalization; this outcome potentially limits therapeutic benefit.

In general, all endovascular MT techniques are accompanied by the risk of periprocedural thrombus fragmentation (PTF) and subsequent downstream embolism, ${ }^{7-9}$ preventing complete recanalization. Because the neurologic outcome of patients with complete (TICI 3) recanalization is significantly better compared with patients with "almost complete" (TICI $2 \mathrm{~b})^{10}$ or incomplete recanalization (TICI 1-2a), ${ }^{11}$ understanding the factors contributing to PTF may prove beneficial in achieving maximal therapeutic benefit. Previous reports demonstrated that thrombus stability $^{12}$ may influence the incidence of PTF, and analyses of cellular thrombus composition have revealed a possible associa- 
tion between thrombus histology and thrombus etiology ${ }^{13,14}$ as well as clinical outcome. ${ }^{15,16}$ Discrepant results in previous studies ${ }^{17}$ might be primarily explained by low patient numbers and the risk of clot fragmentation. The latter may bias the representative character of the analyzed fragment.

While the main cellular components of a thrombus are known to be fibrin-/platelet accumulations (F/P) as well as red (RBC) and white blood cells (WBC), ${ }^{15,17}$ a higher fraction of RBC has been associated with increased rates of successful endovascular recanalization as noninvasively measured by whole-thrombus density $(\mathrm{CT})^{13,18}$ and corresponding blooming artifacts (MR imaging). ${ }^{16,18-21}$ Besides common thrombus characteristics obtained from $H \& E$ staining, new evidence has emerged that the degree of inflammatory cell invasion, particularly by neutrophils, may alter the stability and degradation of a thrombus. ${ }^{22,23}$ This finding is of particular interest because neutrophils exhibit fibrinolytic activity, which may weaken clot stability. ${ }^{24}$ Nevertheless, the impact of inflammatory cells on the mechanical properties of a thrombus remains uncertain, especially in the context of stroke thrombectomy. Potential knowledge of the clot composition before MT may be a further valuable tool to aid in the selection of the most appropriate devices and techniques to avoid PTF.

To this end, this is the first study investigating the dependency of procedural thrombectomy characteristics on anatomic and immune-histochemical thrombus histology, to our knowledge.

\section{MATERIALS AND METHODS Subjects and Outcome}

All consecutive patients presenting with a stroke due to largevessel occlusion in the anterior circulation between July 2010 and September 2012 at a tertiary care center were included in this retrospective single-center study. Parts of this cohort and histologic analyses have been previously published. ${ }^{14,15}$ All patients underwent MT at the Department of Neuroradiology, and thrombus material was preserved. All patients in whom no thrombus material could be retrieved were excluded. Under this premise, 85 patients ( 45 women; median age, 73 years) formed the final study cohort. This study was approved by the local ethics committee at the Klinikum rechts der Isar of the Technical University of Munich, Germany, in accordance with the ethical standards of the 1964 Declaration of Helsinki and its later amendments. ${ }^{25}$ Clinical outcomes were measured as NIHSS at the day of discharge. Substantial neurologic improvement was defined as either NIHSS at day of discharge $\leq 1$ or the difference between NIHSS on admission and NIHSS at the day discharge $\geq 8$. ${ }^{26}$ This definition was based on previous studies that have shown this criterion to be a sensitive outcome measure. ${ }^{26}$

\section{Image Analysis}

The primary occlusion site (POS) was defined as the location and extent of the initial thrombus as evaluated on a synopsis of admission CT/CTA and initial DSA. After recanalization of the POS, any vessel occlusions distal from the POS were considered emboli due to PTF. This simplified definition is based on previous findings, which have shown that $>90 \%$ of intracranial vessel occlusions are caused by a single thrombus. ${ }^{27}$ Hence, most vessel occlusions after POS recanalization are due to PTF. Emboli following PTF were further categorized into proximal, intermediate, and distal, according to their localization in lateral and corresponding anteroposterior DSA projections (On-line Fig 1). Emboli involving the M1 or M2 segment were always defined as proximal emboli, whereas those located distal to the pericallosal artery (on lateral projections) were considered distal emboli (see examples of emboli in Figs 1 and 2). The remaining vessel occlusions were assigned to the group of intermediate emboli. A schematic classification of emboli locations in cases of initial MCA or carotid-T occlusions is presented in On-line Fig 1. The success of POS recanalization (TICI-POS) as well as after additional rescue maneuvers (TICI-FINAL) was rated according to the original TICI scale, with TICI $2 \mathrm{~b}$ defined as reperfusion of more than two-thirds of the initial occluded territory. ${ }^{25}$ Note that TICI-POS and TICI-FINAL may be the same (ie, if no additional maneuvers were performed). All images were evaluated in consensus by 2 experienced neuroradiologists.

\section{Endovascular Procedure}

Patients underwent angiography if groin puncture could be performed within 6 hours after symptom onset, clinical presentation was severe (NIHSS $\geq 4$ ), and no early infarct signs involving more than one-third of the MCA territory were present on cranial CT on admission. IV rtPA was administered as "bridging therapy" in the absence of contraindication $(n=58,68.2 \%)$. All stent-retriever passages were counted, and the number of maneuvers was registered after POS recanalization and at the end of the procedure. All procedures were performed by using a distal access catheter (MCA/anterior cerebral artery [ACA]) or proximal flow arrest by balloon occlusion (carotid-T) and one of the following stent retrievers (maneuvers with the respective device): Solitaire ( $n=45$; Covidien, Irvine, California), pREset $4-20$ ( $n=58$; Phenox, Bochum, Germany), Trevo $(n=29$; Stryker, Kalamazoo, Michigan), Revive ( $n=2$; Codman Neurovascular, Raynham, Massachusetts), Pulse ( $n=4$; Penumbra, Almeda, California), and Separator 3D ( $n=6$, Penumbra). Aspiration techniques, applied as stand-alone approach, were not performed in this study.

\section{Thrombus Histology}

After clot retrieval, thrombus material was fixed in phosphatebuffered $4 \%$ formalin. $\mathrm{H} \& \mathrm{E}$ staining and subsequent quantitative analysis of WBC, RBC, and F/P content were performed as described previously. ${ }^{14,15}$ To assess the prevalence of neutrophils within the thrombus, we stained the samples immunohistochemically by using an anti-neutrophil elastase monoclonal mouse antibody (clone NP57, M0752; Dako Denmark, Glostrup, Denmark). The number of neutrophil elastase (NE)-positive cells was semiquantitatively evaluated by 2 independent raters (NE index) who were blinded to the clinical data. Discrepancies were rated in consensus in a separate session. Evaluation was performed by using a 5-step grading scale: 0 (none), 1 (scattered), 2 (intermediate), 3 (clustered), 4 (high, $>50 \%$ of all cells) (Fig 3 ).

\section{Statistical Analysis and Illustrations}

The Shapiro-Wilk test was applied to analyze data for normal distribution. Because all variables, except NIHSS on admission ( $P=.322$ in Shapiro-Wilk test), were non-normally distributed, 


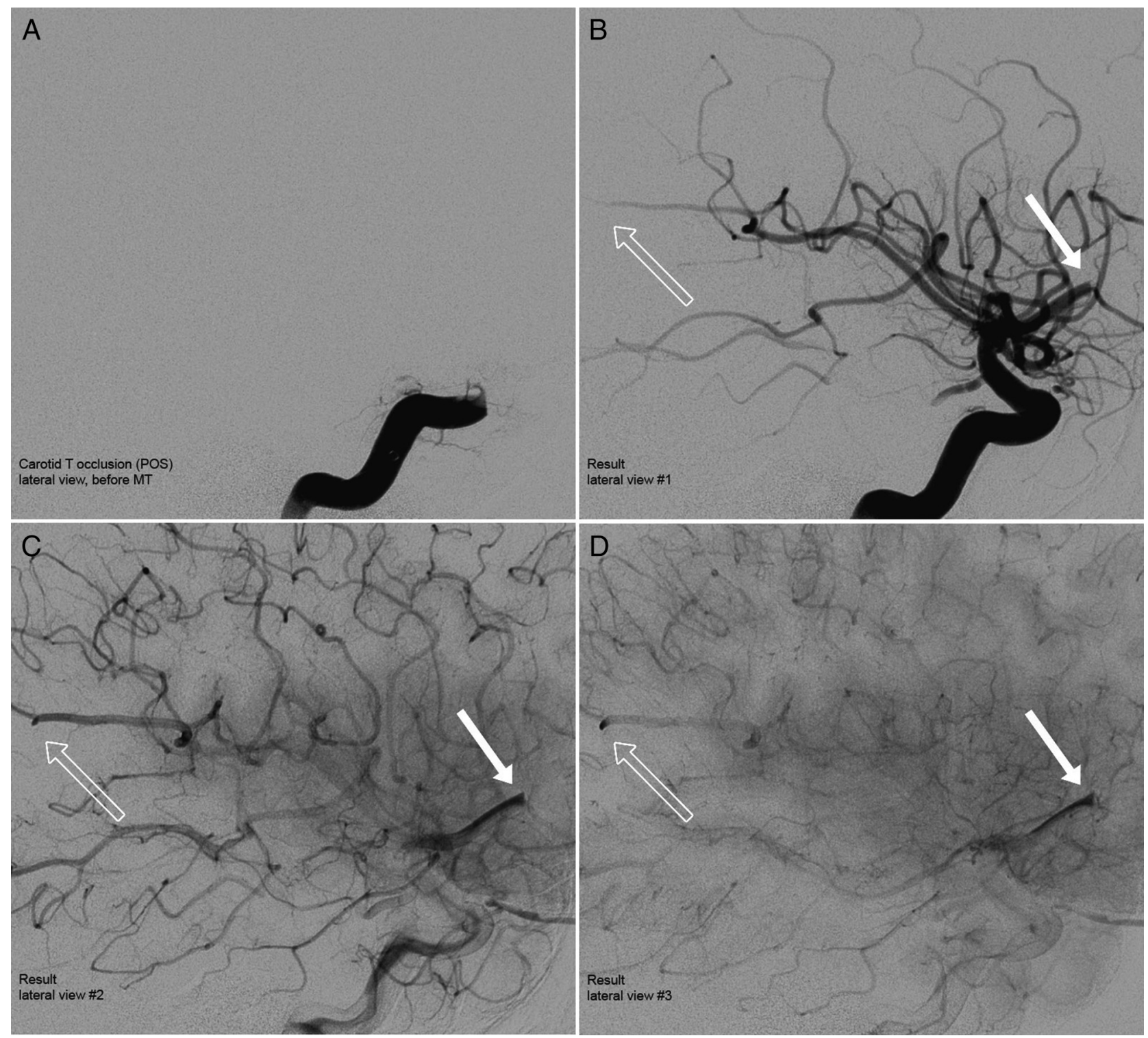

FIG 1. DSA images in a lateral projection in a case of initial carotid-T occlusion (A). Dynamic images (delay $=1$ second) after successful POS recanalization resulting in 1 proximal (filled arrow) and 1 intermediate embolus (open arrow) due to PTF (B-D).

bivariate correlation analysis with the 2-sided Spearman correlation was performed. Frequency counts and median/mean comparison were evaluated by using standard statistical measures (Fisher exact test, Mann-Whitney $U$ test). For median values, the interquartile range (IQR) is shown; for mean values, the SD is shown. Because PTF was shown to be a multifactorial process (eg, age, MCA/ACA versus ICA occlusion, bridging therapy; see "Results"), we adjusted the analysis of histologic clot characteristics for these potential confounders by using a multivariate logistic regression. For statistical analysis, SPSS statistics, release 23.0 (IBM, Armonk, New York), was used. Illustrations were prepared by using Adobe Photoshop CS4 (Adobe Systems, Mountain View, California).

\section{RESULTS}

\section{Study Population and Outcomes}

Inclusion criteria were met by 85 patients (mean age, $70.2 \pm 14.6$ years; 45 women) (Table). Approximately one-third ( $n=26$,
$30.6 \%)$ of patients presented with a carotid-T occlusion, while the rest had isolated occlusions of the MCA/ACA ( $n=57,67.1 \%$, and $n=2,2.3 \%$, respectively). The median NIHSS score at presentation was 15 (IQR, 10.5-18) and improved to 5.5 (IQR, 2-14) by the day of discharge. The median symptom-onset-to-treatmenttime of 225 minutes (IQR, 165-278.75 minutes) could be sufficiently determined for 72 patients. Following recanalization of the POS, 36 patients (42.2\%) showed no peripheral emboli, corresponding to an instant TICI 3 recanalization. Rescue therapy, namely retrieving downstream thrombi due to PTF, was successful in 27 cases. A median of 1 (IQR, 1-3.5) additional stent-retriever maneuver was required to perform the rescue therapy. The final rate of successful recanalization was $91.7 \%$.

When a single device type was used, distribution of final reperfusion success and the number of emboli did not differ among different stent-retriever types $(P=.442$ for final reperfusion success and $P=.931$ for the number of all emboli; On-line Fig 2). Use 

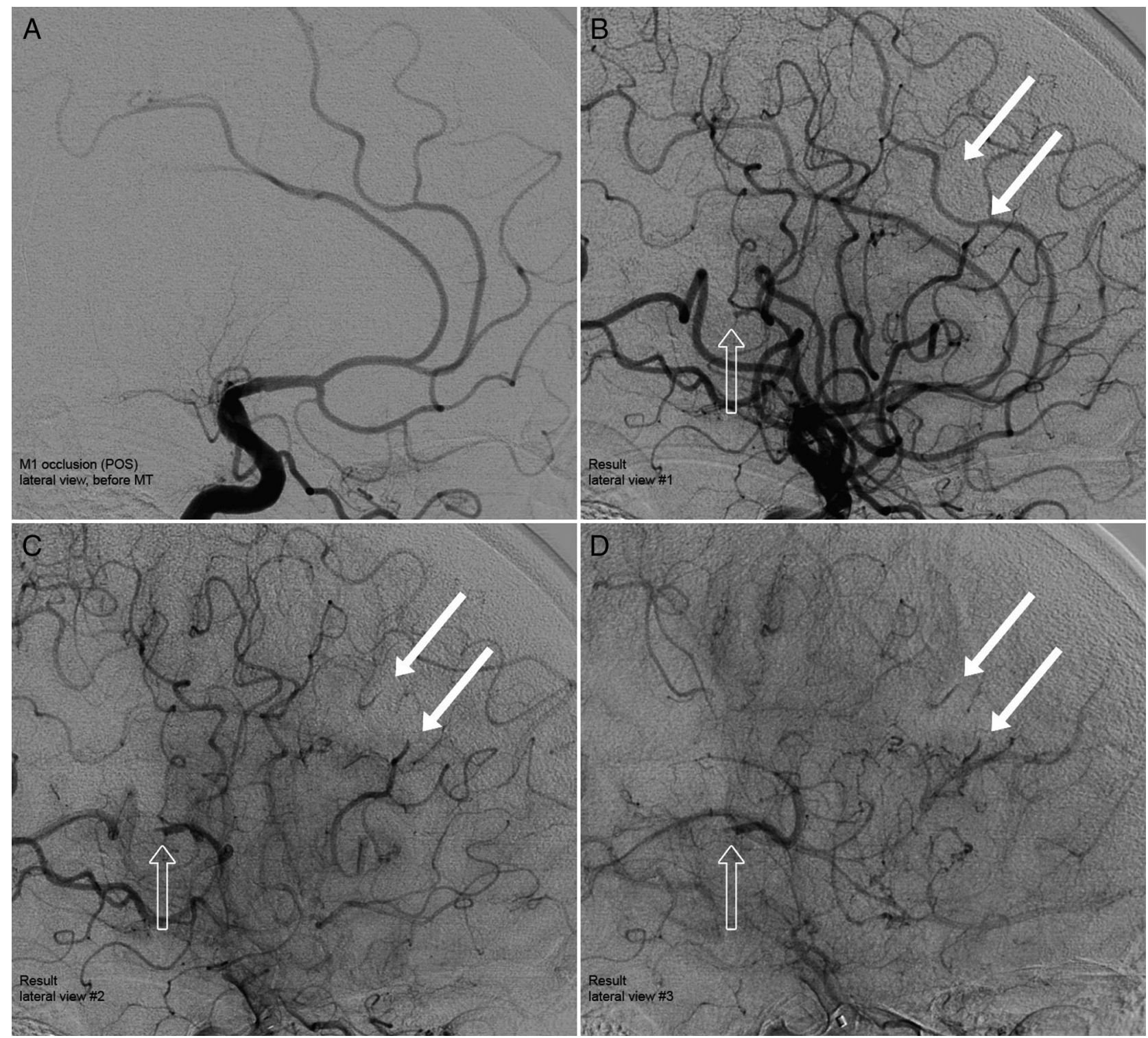

FIG 2. DSA images in a lateral projection in a case of initial $M 1$ occlusion $(A)$. Dynamic images (delay $=1$ second) after successful recanalization of the POS, resulting in 1 intermediate (open arrow) and 2 distal emboli (filled arrows) due to PTF (B-D).

of multiple device types during a solitary thrombectomy was associated with lower rates of successful recanalization $(P=.042)$. Occurrence of proximal and intermediate emboli after recanalization of the POS was associated with poorer neurologic outcome (NIHSS-proximal emboli: Spearman $r, 0.279 ; P=.013$; NIHSSintermediate emboli: Spearman $r, 0.223 ; P=.049$ ), whereas patients showing a substantial neurologic improvement had fewer proximal $(P=.029)$ and intermediate emboli $(P=.018)$.

\section{Physical Clot Properties}

A consistent, inverse trend could be observed between the ease of the procedure and the risk of PTF: An easier procedure, namely requiring fewer stent-retriever maneuvers and less time to POS recanalization, was associated with a higher risk of distal and intermediate embolization (On-line Table). We also noted an age dependency of emboli occurrence because younger patients were at higher risk for PTF, resulting in higher numbers of intermediate and overall thrombus fragments distal to the POS. No corre- lation could be found between the time from symptom onset to groin puncture and the risk of embolization or maneuvers required. Administration of preinterventional IV rtPA tended to be associated with fewer endovascular maneuvers (2 versus $3, P=$ .054 ), shorter time to POS recanalization (30 minutes versus 47 minutes, $P=.051$ ), but a higher sum of overall emboli ( 1 versus 0 , $P=.067)$. In carotid-T occlusions as opposed to isolated MCA/ ACA occlusions, more device passages were required to retrieve the primary thrombus ( 3 versus $2, P=.006$ ), resulting in longer times for POS recanalization (66 versus 33 minutes, $P<.001$ ). However, no difference could be found regarding the location or number of occurring emboli (all $P>.5$ ).

\section{Thrombus Histology}

The mean fraction of RBC, F/P, and WBC was $42 \%$ (IQR, 22\%$57 \%$ ), 49\% (IQR, 36\%-69\%), and 7\% (IQR, 5\%-11\%), respectively. The median NE index, as determined for 42 patients, was 2 (IQR, 1-3). Clot histology characteristics did not differ between 

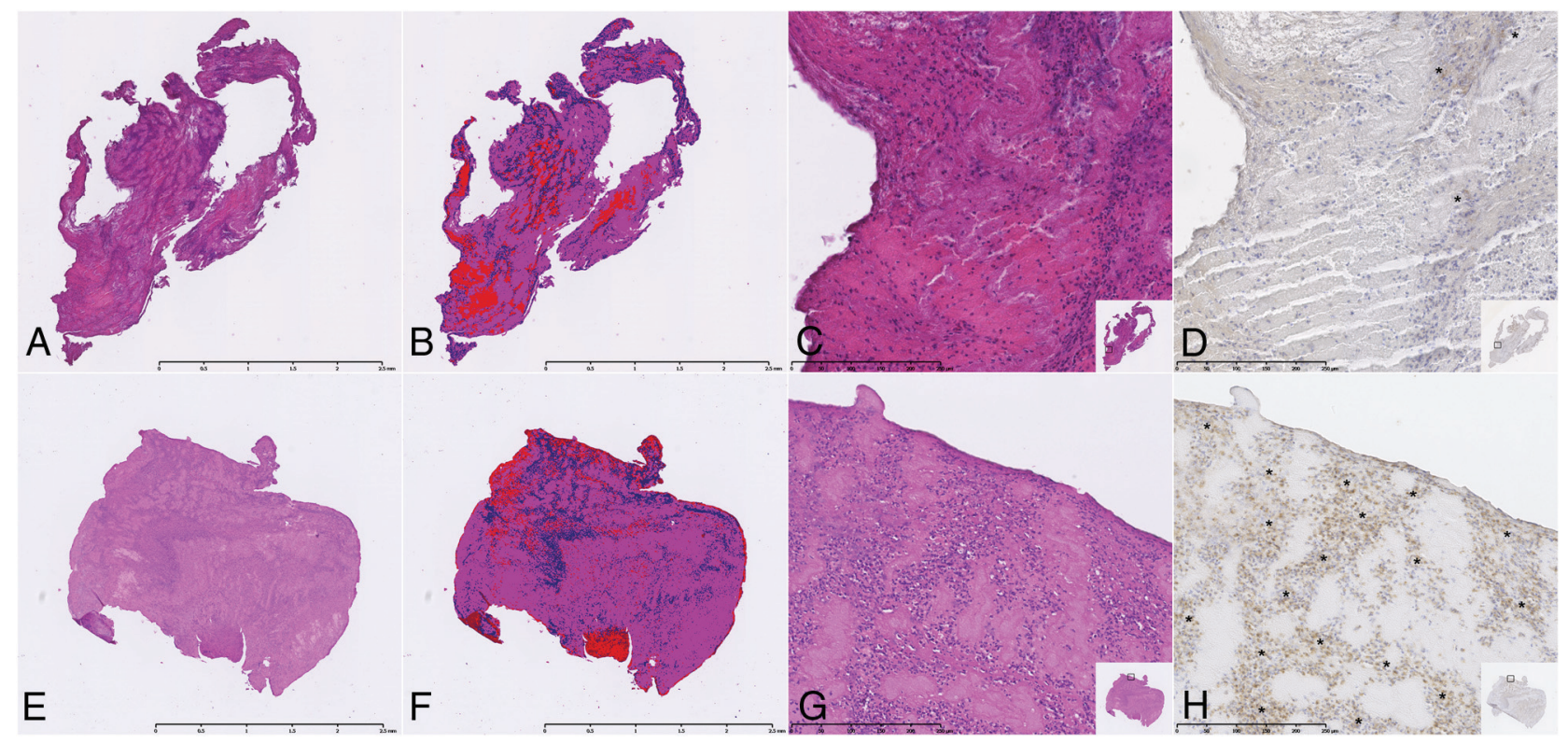

FIG 3. Histologic specimens of 2 different clots with H\&E staining (magnification $\times 30)(A$ and $E)$, segmentation of RBC (red), $F / P(p u r p l e), W B C$ (blue), and staining artifacts (brown) for composite quantification ( $B$ and F). H\&E staining (magnification $\times 200)(C$ and $G)$ and NE staining (magnification $\times 200)(D$ and $H$ ) with accumulation of $\mathrm{NE}$-positive cells marked (asterisks). Clot composition is the following: upper row: $30 \%$ RBC, $51 \%$ F/P, 19\% WBC; NE index 1; lower row: 7\% RBC, 84\% F/P, 9\% WBC, NE index 4.

Patient characteristics, clot histology, and procedural parameters

\begin{tabular}{lc}
\hline & $\begin{array}{c}\text { No. or Median with } \\
\text { Interquartile Range }\end{array}$ \\
\hline Patients (No.) & 85 \\
Male/female (No.) & $40 / 45(47 \% / 53 \%)$ \\
Age (median) (yr) & $73(65-80)$ \\
POS (No.) & \\
ICA/carotid-T & $26(30.6 \%)$ \\
ACA & $2(2.3 \%)$ \\
MCA & $57(67.1 \%)$ \\
IV rtPA bridging therapy (No.) & $58(68 \%)$ \\
Maneuvers (No.) & $2(1-4)$ \\
Procedure time for POS recanalization (min) & $37(21-64)$ \\
TICl after POS recanalization (No.) & \\
1 & $1(1 \%)$ \\
$2 a$ & $27(32 \%)$ \\
$2 b$ & $21(25 \%)$ \\
3 & $36(42 \%)$ \\
Occlusions due to PTF ( $n=101)$ & \\
Proximal & $26(26 \%)$ \\
Intermediate & $50(50 \%)$ \\
Distal & $25(25 \%)$ \\
Clot histology (fraction in \%) & \\
RBC & $37(27-48)$ \\
F/P & $57(47-67)$ \\
WBC & $5(4-7)$ \\
\hline
\end{tabular}

patients receiving bridging therapy and patients who did not (all, $P>$.3). Except for a higher NE index in carotid-T thrombi (median 2; IQR, 2-3.5, versus 2; IQR, 1-2; $P=.008)$, no difference in histologic clot characteristics could be found when comparing MCA and carotid-T occlusions (all, $P>.3$ ). We could not observe a correlation between the time from symptom onset to recanalization or age and fraction of RBC, F/P, and WBC (all, $P>.5$ ). No consistent association between procedural parameters and $\mathrm{RBC}$, $\mathrm{F} / \mathrm{P}$, or WBC content within the clot could be found (all, $P>.1$;
On-line Table). This was also true when restricting analysis to different stent-retriever types (all, $P>.2$ ). However, the fraction of RBC (median RBC fraction, 50\% [IQR, 28\%-59\%], versus $39 \%$ [IQR, $21 \%-57 \%$ ]; $P=.183$ ) and the degree of neutrophil invasion (median NE index, 2 [IQR, 2-3] versus 2 [IQR, 1-3]; $P=$ .192) tended to be higher in patients with multiple embolizations. The effect of neutrophil invasion on the occurrence of multiple emboli remained statistically tangible (without case restriction) when adjusting for age, site of occlusion (carotid-T versus MCA/ACA), and bridging therapy in a multivariate logistic regression model with multiple $(>1)$ emboli defined as dependent variables (adjusted OR, 4.6; 95\% CI, 1.1-19.7 for every NE index grade increase; $P=.041$; pseudo- $R^{2}=0.568$ ). In contrast, the fraction of $\mathrm{RBC}$ was not a significant factor associated with the occurrence of multiple emboli by using the same logistic regression model (OR, 1.0; 95\% CI, $0.9-1.0 ; P=.390)$. Pursuant to the first finding, the median NE index was lower in successfully recanalized patients (2 versus 3; $P=.033$, On-line Fig 3). Furthermore, the NE index was an independent predictor of unsuccessful recanalization (TICI-FINAL $<2 \mathrm{~b}$, [see above for the logistic regression used]; adjusted OR, 0.3; 95\% CI, 0.1-0.9; $P=.050$; pseudo- $R 2=0.322)$.

\section{DISCUSSION}

PTF during MT in acute stroke is common and was observed in more than half of the patients. This study shows 3 major findings: 1) In vivo clots differ regarding their MT-relevant mechanical properties, 2) common histologic clot characteristics do not consistently correlate with the mechanical clot properties, and 3) neutrophil invasion of the clot is linked to PTF and might serve as a novel surrogate for clot stability.

Our results show that easily retractable clots are generally prone to small/intermediate fragment dissociation, while those retracted with considerable effort are usually more stable and less 
susceptible to PTF. During endovascular stroke treatment, the use of stent retrievers and aspiration devices has a risk of thrombus fragmentation, which can lead to an "embolic shower." 28,29 Smaller fragments $(<200 \mu \mathrm{m})$ constitute, by far, the highest percentage of occurring emboli. ${ }^{28}$ They usually dissolve due to spontaneous thrombolysis or revascularization as achieved by embolus extravasation. ${ }^{30}$ However, those emboli are usually undetected by conventional DSA, and their true clinical impact remains unclear. ${ }^{31}$ Larger emboli $(>200 \mu \mathrm{m})$ cause clinically relevant cerebral occlusion $^{32}$ and lead to angiographically defined incomplete recanalization (TICI $<3$ ). We have found those emboli to be clinically important because intermediate and proximal emboli were associated with lower NIHSS scores at discharge. Occurrence and "rescue" removal of those emboli are of important clinical relevance because patients with complete recanalization have fewer neurologic deficits. ${ }^{10}$

The beneficial effects of preinterventional systemic rtPA administration concur with our assessment; this finding suggests a facilitating effect of bridging therapy. ${ }^{33-35}$ Patients receiving rtPA needed fewer maneuvers and had shorter procedure times. However, the presumed bridging therapy-related thrombus softening came with the risk of PTF, which has been reported previously. ${ }^{36}$

Thrombus characteristics have been shown to predict technical outcome in endovascular stroke therapy. Particularly, thrombi with higher Hounsfield units are associated with higher rates of successful recanalization. ${ }^{19,21}$ Given prior histologic analyses, it seems reasonable to assume that those thrombi are $\mathrm{RBC}$ rich and have low F/P content. ${ }^{18}$ In the present study, thrombi prone to dissociate into multiple emboli tended to have a higher RBC fraction. However, this association was inconsistent and statistically irrelevant when correcting for possible confounders. Our data regarding the true success rate of thrombectomy are particularly limited because we only analyzed cases in which thrombus material could be retrieved (usually TICI $>1-2 a$ ). Hence, the true impact of RBC on the capability of retrieving the clot and POS recanalization cannot finally be assessed in the presented cohort.

To the best of our knowledge, this is the first study that establishes a possible link between procedurally relevant thrombus stability and the content of neutrophils. Neutrophils invade newly formed thrombi through recruitment due to adherent activated platelets. ${ }^{37}$ Adhesion triggers activation of neutrophils and subsequent release of neutrophil elastase. ${ }^{38}$ Initially, proinflammatory mediators promote fibrin cross-linking and the formation of neutrophil extracellular traps, which promote a procoagulatory state. ${ }^{39}$ However, activated neutrophils in the later phase also seem to restrict growth and promote thrombus degradation by NE-dependent fibrinolysis ("cell-dependent thrombolysis"). ${ }^{22}$ Komorowicz et $\mathrm{al}^{24}$ could indeed show that NE triggers the release of thrombus fragments in the soluble phase when incubated under shear stress conditions. Beyond altering the stability properties of thrombi, experimental data further suggest that NE seems to be involved in inflammatory reperfusion damage, ${ }^{40}$ and a recent study proposed an association between the presence of $\mathrm{CD} 4^{+}$ T-cells and $\mathrm{CD} 68^{+}$-monocytes/macrophages within the thrombus and stroke severity. ${ }^{41}$ However, the true impact of "thromboinflammation" has yet to be evaluated in further studies. ${ }^{42}$ In this context, the presented data underscore the need for consid- ering and correcting for potential effects of inflammatory cells on thrombus stability, when evaluating its effect on clinical outcome in patients with stroke who were endovascularly treated.

Our study has several limitations beyond the common ones of a retrospective study design. Due to our inclusion criteria, the cohort exhibits a selection bias for successfully recanalized patients because histologic analysis was feasible only in cases in which the thrombus could be retrieved. Thus, results regarding the effects of histologic parameters on the true success rates of MT should be interpreted cautiously. Second, assessing the rate and location of emboli by using midprocedural DSA is a simplification and might not be as sensitive as susceptibility-weighted imaging for thrombus detection. ${ }^{43}$ However, it is the only feasible method of PTF detection during the procedure and has the advantage of assessing the hemodynamic relevance of emboli due to PTF. Third, this technique is not capable of differentiating periand preprocedural thrombus fragmentation (distal to the POS). However, because previous studies have found a low frequency of preprocedural fragmentation $(<10 \%),{ }^{27,43}$ the rate of false-positive classification is expected to be rather low. Fourth, the grading of the NE of the clot is based on a semiquantitative scale and was performed visually. Subjective analysis may, in the future, be overcome by ensuring comparable staining intensity and following threshold-based software quantification (comparable with the algorithm used for the analysis of H\&E staining). Fifth, because we analyzed the thrombus retrieved from the POS, the histologic characteristics of the fragments causing distal emboli remain uncertain and might differ from the clot composition of the retrieved thrombus. One may speculate that dissolvable tissue islands or additional apposition thrombi might accumulate at the clot margins. These areas may be more prone to fragmenting during endovascular manipulation than the main thrombus and might show a different histology. Finally, because different stent retrievers were used, recanalization success and subsequent risk of PTF might be influenced by the choice of the device.

\section{CONCLUSIONS}

Younger age, easy-to-retrieve thrombi, and bridging thrombolysis are factors that may have a higher risk of PTF. Conventional histologic characteristics (RBC, WBC, F/P) do not seem to influence MT-relevant thrombus stability. However, higher amounts of clot-infiltrating neutrophils were related to an increased risk of PTF. This observation aligns well with the proposed thrombolytic capacity of neutrophilic enzymes and may implicate them as a novel marker for clot stability, which should be addressed in future clot studies.

Disclosures: Claus Zimmer-UNRELATED: Board Membership: Philips Healthcare and Bayer Schering Pharma, Comments: scientific advisory boards*; Payment for Lectures including Service on Speakers Bureaus: Bayer Schering Pharma and Philips Healthcare, Comments: speaker honoraria; Other: Biogen Idec, Quintiles, MSD Sharp \& Dome, Boehringer Ingelheim, Inventive Health Clinical UK Ltd, advanceCOR, BrainsGate, Pfizer, Bayer Schering Pharma, Novartis, Roche, Servier, Penumbra, WCT $\mathrm{GmbH}$, Syngis, SSS International Clinical Research, PPD Germany GmbH, Worldwide Clinical Trials Ltd, phenox, Covidien, Actelion, Medivation, Medtronic, Harrison Clinical Research, Concentric, pharmtrace, Reverse Medical Corp, Premier Research Germany Ltd, Surpass Medical Ltd, and GlaxoSmithKline, Comments: research support and investigator fees for clinical studies.* Lucas Schirmer-UNRELATED: Patents (Planned, Pending, or Issued): WO2015166057A1; Comments: I filed a patent for the detection of antibodies against KIR4.1 in a subpopulation of patients with MS; the 
patent has been published, however, payments have not been made yet; Travel/ Accommodations/Meeting Expenses Unrelated to Activities Listed: Genzyme, Comments: travel support. Holger Poppert-UNRELATED: Consultancy: Bayer HealthCare, Daiichi Sankyo Pharmaceuticals; Grants/Grants Pending: Deutsche Stiftung Neurologie*; Payment for Lectures including Service on Speakers Bureaus: Bayer HealthCare, Daiichi Sankyo Pharmaceuticals, Boehringer Ingelheim, BristolMyers Squibb, Pfizer, DEGUM, RG Gesellschaft für Information und Organisation, Medkom Akademie, CVA-Cardio Vascular Academy gemeinnützige $\mathrm{GmbH}$. Thomas Huber-UNRELATED: Consultancy: Brainlab, Feldkirchen, Germany, Comments: consultancy for atlas-based segmentation techniques. *Money paid to the institution.

\section{REFERENCES}

1. Berkhemer OA, Fransen PS, Beumer D, et al. A randomized trial of intraarterial treatment for acute ischemic stroke. N Engl JMed 2015; 372:11-20 CrossRef Medline

2. Goyal M, Demchuk AM, Menon BK, et al; ESCAPE Trial Investigators. Randomized assessment of rapid endovascular treatment of ischemic stroke. $N$ Engl J Med 2015;372:1019-30 CrossRef Medline

3. Jovin TG, Chamorro A, Cobo E, et al; REVASCAT Trial Investigators. Thrombectomy within $\mathbf{8}$ hours after symptom onset in ischemic stroke. N Engl J Med 2015;372:2296-306 CrossRef Medline

4. Campbell BC, Mitchell PJ, Kleinig TJ, et al; EXTEND-IA Investigators. Endovascular therapy for ischemic stroke with perfusion-imaging selection. $N$ Engl J Med 2015;372:1009-18 CrossRef Medline

5. Saver JL, Goyal M, Bonafe A, et al; SWIFT PRIME Investigators. Stent-retriever thrombectomy after intravenous t-PA vs. t-PA alone in stroke. $N$ Engl J Med 2015;372:2285-95 CrossRef Medline

6. Manning NW, Chapot R, Meyers PM. Endovascular stroke management: key elements of success. Cerebrovasc Dis 2016;42: 170-77 CrossRef Medline

7. Gralla J, Schroth G, Remonda L, et al. Mechanical thrombectomy for acute ischemic stroke: thrombus-device interaction, efficiency, and complications in vivo. Stroke 2006;37:3019-24 CrossRef Medline

8. Gralla J, Schroth $\mathrm{G}$, Remonda $\mathrm{L}$, et al. A dedicated animal model for mechanical thrombectomy in acute stroke. AJNR Am J Neuroradiol 2006;27:1357-61 Medline

9. Penumbra Pivotal Stroke Trial Investigators. The Penumbra Pivotal Stroke Trial: safety and effectiveness of a new generation of mechanical devices for clot removal in intracranial large vessel occlusive disease. Stroke 2009;40:2761-68 CrossRef Medline

10. Kleine JF, Wunderlich S, Zimmer C, et al. Time to redefine success? TICI 3 versus TICI $2 b$ recanalization in middle cerebral artery occlusion treated with thrombectomy. J Neurointerv Surg 2016 Feb 17. [Epub ahead of print] Medline

11. Jayaraman MV, Grossberg JA, Meisel KM, et al. The clinical and radiographic importance of distinguishing partial from near-complete reperfusion following intra-arterial stroke therapy. $A J N R$ Am J Neuroradiol 2013;34:135-39 CrossRef Medline

12. Machi P, Jourdan F, Ambard D, et al. Experimental evaluation of stent retrievers' mechanical properties and effectiveness. J Neurointerv Surg 2016 Mar 25. [Epub ahead of print] CrossRef Medline

13. Niesten JM, van der Schaaf IC, van Dam L, et al. Histopathologic composition of cerebral thrombi of acute stroke patients is correlated with stroke subtype and thrombus attenuation. PLoS One 2014;9:e88882 CrossRef Medline

14. Boeckh-Behrens T, Kleine JF, Zimmer C, et al. Thrombus histology suggests cardioembolic cause in cryptogenic stroke. Stroke 2016;47: 1864-71 CrossRef Medline

15. Boeckh-Behrens T, Schubert M, Förschler A, et al. The impact of histological clot composition in embolic stroke. Clin Neuroradiol 2016;26:189-97 CrossRef Medline

16. Kim SK, Yoon W, Kim TS, et al. Histologic analysis of retrieved clots in acute ischemic stroke: correlation with stroke etiology and gradient-echo MRI. AJNR Am J Neuroradiol 2015;36:1756-62 CrossRef Medline

17. Marder VJ, Chute DJ, Starkman S, et al. Analysis of thrombi retrieved from cerebral arteries of patients with acute ischemic stroke. Stroke 2006;37:2086-93 CrossRef Medline
18. Liebeskind DS, Sanossian N, Yong WH, et al. CT and MRI early vessel signs reflect clot composition in acute stroke. Stroke 2011;42: 1237-43 CrossRef Medline

19. Mokin M, Morr S, Natarajan SK, et al. Thrombus density predicts successful recanalization with Solitaire stent retriever thrombectomy in acute ischemic stroke. J Neurointerv Surg 2015;7:104-07 CrossRef Medline

20. Froehler MT, Tateshima S, Duckwiler G, et al; UCLA Stroke Investigators. The hyperdense vessel sign on CT predicts successful recanalization with the Merci device in acute ischemic stroke. J Neurointerv Surg 2013;5:289-93 CrossRef Medline

21. Moftakhar P, English JD, Cooke DL, et al. Density of thrombus on admission CT predicts revascularization efficacy in large vessel occlusion acute ischemic stroke. Stroke 2013;44:243-45 CrossRef Medline

22. Imamura $T$, Kaneda $H$, Nakamura S. New functions of neutrophils in the arthus reaction: expression of tissue factor, the clotting initiator, and fibrinolysis by elastase. Lab Invest 2002;82:1287-95 CrossRef Medline

23. Michel JB, Ho-Tin-Noé B. Thrombi and neutrophils. Circ Res 2015; 116:1107-08 CrossRef Medline

24. Komorowicz E, Kolev K, Léránt I, et al. Flow rate-modulated dissolution of fibrin with clot-embedded and circulating proteases. Circ Res 1998;82:1102-08 CrossRef Medline

25. Higashida RT, Furlan AJ, Roberts H, et al; Technology Assessment Committee of the American Society of Interventional and Therapeutic Neuroradiology, Technology Assessment Committee of the Society of Interventional Radiology. Trial design and reporting standards for intra-arterial cerebral thrombolysis for acute ischemic stroke. Stroke 2003;34:e109-37 CrossRef Medline

26. Kerr DM, Fulton RL, Lees KR, et al; VISTA Collaborators. Seven-day NIHSS is a sensitive outcome measure for exploratory clinical trials in acute stroke: evidence from the Virtual International Stroke Trials Archive. Stroke 2012;43:1401-03 CrossRef Medline

27. Gratz PP, Schroth G, Gralla J, et al. Whole-brain susceptibilityweighted thrombus imaging in stroke: fragmented thrombi predict worse outcome. AJNR Am J Neuroradiol 2015;36:1277-82 CrossRef Medline

28. Chueh JY, Kühn AL, Puri AS, et al. Reduction in distal emboli with proximal flow control during mechanical thrombectomy: a quantitative in vitro study. Stroke 2013;44:1396-401 CrossRef Medline

29. Gobin YP, Starkman S, Duckwiler GR, et al. MERCI 1: a phase 1 study of Mechanical Embolus Removal in Cerebral Ischemia. Stroke 2004; 35:2848-54 CrossRef Medline

30. Yoo AJ, Simonsen CZ, Prabhakaran S, et al; Cerebral Angiographic Revascularization Grading Collaborators. Refining angiographic biomarkers of revascularization: improving outcome prediction after intra-arterial therapy. Stroke 2013;44:2509-12 CrossRef Medline

31. Rapp JH, Pan XM, Sharp FR, et al. Atheroemboli to the brain: size threshold for causing acute neuronal cell death. J Vasc Surg 2000;32: 68-76 CrossRef Medline

32. Sorimachi T, Nishino K, Shimbo J, et al. Routine use of debris aspiration before retrieval of distal filter protection devices in carotid arterial stenting: analysis of captured debris and evaluation of clinical results. Neurosurgery 2010;67:1260-67; discussion 1267 CrossRef Medline

33. Behme D, Kabbasch C, Kowoll A, et al. Intravenous thrombolysis facilitates successful recanalization with stent-retriever mechanical thrombectomy in middle cerebral artery occlusions. J Stroke Cerebrovasc Dis 2016;25:954-59 CrossRef Medline

34. Leker RR, Pikis S, Gomori JM, et al. Is bridging necessary? A pilot study of bridging versus primary Stentriever-based endovascular reperfusion in large anterior circulation strokes. J Stroke Cerebrovasc Dis 2015;24:1163-67 CrossRef Medline

35. Pfefferkorn T, Holtmannspötter M, Patzig M, et al. Preceding intravenous thrombolysis facilitates endovascular mechanical recanalization in large intracranial artery occlusion. Int J Stroke 2012;7: 14-18 CrossRef Medline 
36. Kaesmacher J, Kleine JF. Bridging therapy with i. v. rtPA in MCA occlusion prior to endovascular thrombectomy: a double-edged sword? Clin Neuroradiol 2016 Aug 19. [Epub ahead of print] Medline

37. Kirchhofer D, Riederer MA, Baumgartner HR. Specific accumulation of circulating monocytes and polymorphonuclear leukocytes on platelet thrombi in a vascular injury model. Blood 1997;89: 1270-78 Medline

38. Rainger GE, Rowley AF, Nash GB. Adhesion-dependent release of elastase from human neutrophils in a novel, flow-based model: specificity of different chemotactic agents. Blood 1998;92:4819-27 Medline

39. Massberg S, Grahl L, von Bruehl ML, et al. Reciprocal coupling of coagulation and innate immunity via neutrophil serine proteases. Nat Med 2010;16:887-96 CrossRef Medline
40. Nicolini FA, Mehta JL, Nichols WW, et al. Leukocyte elastase inhibition and t-PA-induced coronary artery thrombolysis in dogs: beneficial effects on myocardial histology. Am Heart J 1991;122: 1245-51 CrossRef Medline

41. Schuhmann MK, Gunreben I, Kleinschnitz C, et al. Immunohistochemical analysis of cerebral thrombi retrieved by mechanical thrombectomy from patients with acute ischemic stroke. Int J Mol Sci 2016;17:298 CrossRef Medline

42. De Meyer SF, Denorme F, Langhauser F, et al. Thromboinflammation in stroke brain damage. Stroke 2016;47:1165-72 CrossRef Medline

43. Klinger-Gratz PP, Schroth G, Gralla J, et al. Protected stent retriever thrombectomy prevents iatrogenic emboli in new vascular territories. Neuroradiology 2015;57:1045-54 CrossRef Medline 